\title{
DETECTION OF SKIN DISEASE IN PERVASIVE HEALTH CARE USING CONVOLUTION NEURAL NETWORK
}

Sarojini Sharon R.K', Sruthy Simon', Rajalakshmi Shenbaga Moorthy', and

\author{
Dr.P.Pabitha \\ 'St.Joseph's Institute of Technology \\ 1sharon110699@gmail.com \\ ${ }^{1}$ sruthysimon451@gmail.com \\ Madras Institute of Technology
}

\begin{abstract}
Abstrac.This paper is about detecting skin disease using convolutional neural network in pervasive health care. Various optimizers in keras model are compared and the optimizer with the highest accuracy percentage is employed to predict skin disease through abnormalities in skin images. Detecting skin diseases by viewing images of skin can be an advancement to a great extent. There are many algorithms in machine language which would help to serve the above mentioned scenario. One of the most efficient algorithms that is being used here is convolutional neural networks. It simplifies the input image by reducing its dimensionality which makes prediction of patterns much easier.Convolutional neural network is used along with keras using tensorflow as backend which enables higher efficiency and accuracy.
\end{abstract}

Keywords: CNN, Keras, Pervasive Healthcare

\section{Introduction}

An optimizer is one of the two arguments which is being used for compiling a Keras model. One can either instantiate an optimizer before passing it to model.compile(), or you can pass it by its string identifier. In the latter case, the default parameters for the optimizer will be used. After comparing various optimizers that will best suit our prediction system, we are employing the optimizer with high accuracy percentage in our prediction model. Detecting various forms of disease through abnormalities in cell images has turned out to be a great fact of advantage especially for people living in remote areas, where there are difficulties in maintaining healthcare centers. In this way people could just take pictures of their infectected cell region and upload it on cloud where doctors will be able to access the cell images and find out if the particular cell region is affected or not. The basic idea that is being used in this paper is medical image analytics of cell mages aiming at improving pervasive healthcare measures. In 
this convolutional neural network is used to detect if a skin disease is present or not .Skin diseases are quite a common problem which is faced commonly by people throughout the world. A quick and comfortable method which has high performance is required to differentiate disease affected skin cells from nondisease affected skin cells. Methods that are Computer- aided, can significantly improve reproducibility and have attracted a great amount of interest in recent literature reviews. In the past few years, many efforts have been taken for automated diagnosis of skin disease detection and also segmentation. In this review, we provide an whole summary of the recent state of sin disease diagnosis and detection. This stated method is unique from other methods in simplicity of the approach developed and also in the type of algorithm used to detect if the skin disease is present or not.

CNN predictor is used to predict if a skin disease is present or not. Generally skin disease does not affect the mortality rate but in some worst cases it definitely does affect the lives of people. Hence it is very necessary to predict if skin disease is present or not in human beings especially people from rural areas, where there are limited medical facilities.

\section{Related Works}

In [1] the skin disease detection system is an automated system that will assist the medical community to identify skin diseases early on. This early detection of the disease is emphasized in order to enable dermatologists to increase the time of diagnosis and the precision of their invention. This approach can also be evaluated on a broad dataset as a future mission. In [2] a analysis of modern techniques based on complex mathematical symmetry for the detection and identification of malaria parasites in photos of stained blood smears. In [10] To discover various forms of dermatological skin diseases, they have used machine sight-based techniques and suggested methods. For training and testing purposes, different types of image processing algorithms for feature extraction and artificial neural networks are used. The system operates in two stages: first pre-process, and then disease detection. With a precision rate of ninety percent, the device successfully identifies 9 different forms of skin diseases. In [3], a ResNet-152 architecture is used, where over 3,797 images have been trained, later increased by a factor of 29 times, using transformations in location, size, and lighting. Finally, the network was tested with 956 images and an area under the curve (AUC) of 0.96 for Melanoma and 0.91 for Basal Cell Carcinoma was reached. In [5] the proposed system provides an effective solution for the identification of skin disease providing an efficiency ranging eighty percent. For Future, accurate detection with secured result analysis can be found out using multiple methods.In [4] a skin disease detection system which is automatic is being proposed. It is useful in the 
medical field for detecting skin diseases early. This system can also be checked for a wide variety of datasets which in turn will enhance the effectiveness of the algorithm. In [6], various non-invasive techniques have been examined for skin cancer classification and detection. This survey concentrates on unique strategies like Genetic Algorithm, SVM, CNN, ABCD rule. In [11] a threshold connected algorithmic rule that acknowledges skin image exploitation in the RGB-HSV-YCbCr model is shown.

In [7] A dual approach that integrates computer vision and machine learning effectively based on the clinically evaluated attributes to identify specifically the disease. The system created an accuracy following training and testing for the six diseases of about ninety percent. So can obtain a lot of information from the data concealed in the images. Using data mining techniques the extraction process can be done. A framework for mining and processing image data based on the decision tree is used. This system [8] improves the classification process to be more accurate. In [9], they have studied the problem of skin disease detection. They have constructed a detection system based on image processing and data mining. A system for the detection of skin diseases that will assist the medical society in the early detection of all types of skin diseases is found. Using histograms, the various features of the enhanced images were segmented. The infected area is identified based on the findings, and skin diseases are found. In [12] shows an overview of different skin sickness. A full report of different skin infections is being examined. The solution to six common skin diseases is given by the proposed technique. So before going to the hospital, the patient should gain prior information. This can also be useful in rural areas where there is no access to a dermatologist. This is a way of detecting skin diseases. Therefore this approach is very simple to adopt and can be used by all sorts of individuals. Here from the data collection, the disease is detected, then compared with a large number of photos of various types of diseases, and finally projected. It was compared with six types of diseases in this work. It can be applied to several other ways in the future.

The research[13] will provide an opportunity to user for finding the skin disease based on symptoms. In this research the data processing of sick people is by using $\mathrm{KNN}$. It aims to learn ordered representations of data by using KNN. In a skin disease detection system, images has to be processed and analysed . In this paper, the KNN algorithms were applied to infected skin images of humans in terms of skin image detection, image processing, and image recognition and image classification.In [14] By allowing a guided visual exploration of the search space through a method called visual analytics for medical image retrieval, they found a new approach to the medical field (VAMIR). The visual analytics approach facilitates the exploration of the entire dataset as a point-of-reference using the query image. In [15] the subsequent things are being proposed 1) it provides comprehensive data on the epidemiology of the most typical skin disorders during a developing country environment, 2) documents their health importance, 3) 
describes measures that would be wont to control them, and 4) permits a rational consideration of the matter. The study was performed with a view to future integration of matters concerning skin diseases in children. In [16][20], Three forms of skin diseases such as herpes, dermatitis, and psoriasis are recognized through a modern recognition system. Skin images have been preprocessed by filtering and modifying to strip out noise and irrelevant characteristics. The greylevel co-occurrence matrix (GLCM) approach was then used to segment images of skin disease. It was possible to correctly obtain the texture and color characteristics of various skin disease photos. Finally, three forms of skin diseases were described using the SVM characterization process. In [17] [19] A new approach for studying artificial neural networks is proposed to gain insight into their internal functionality. In terms of differential operators and with various angles of action, the components of a feed forward-back propagation neural network that has been trained to locate edges in images are objectified.

\section{System Architecture}

The system architecture for the pervasive health care system based on Convolutional Neural Netowrk (CNN) is shown in Figure 1. The person at remote submits the image data of the skin to the health care professional. CNN predictor has been trained and tested in cloud. The health care professional submits the user request to the $\mathrm{CNN}$ predictor and the $\mathrm{CNN}$ predictor returns the result which is then issued to the concerned person.

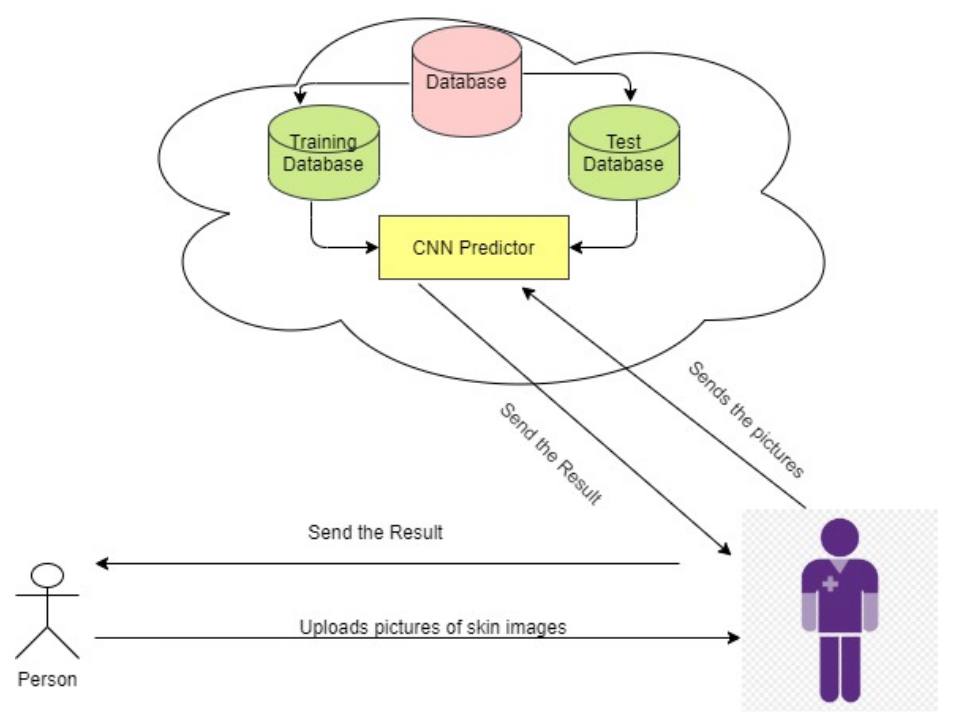

Figure 1: Architectural Diagram of the proposed pervasive skin disease detection 


\section{Implementation}

Convolutional neural networks (CNNs) are adequately used for the tasks of recognition of images and classification. For example, to find out whether a skin disease is present in each of the images or not. CNNs process images from the top. Neurons that are placed earlier in the network are responsible for inspecting small windows of pixels and finding simple, small features such as edges and corners. These outputs are then put into neurons in the intermediate layers, which search for larger features. This next set of outputs are used to make a final judgment about whether the image contains a skin disease or not. The schematic diagram of Convolutional Neural Network is shown in Figure 2.

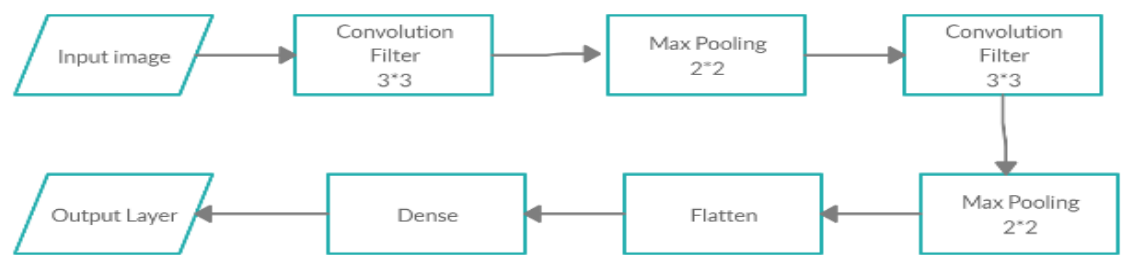

Fig 2: Convolutional Neural Network

The Convolution layer recieves an image as input with $\mathbf{m * n}(\mathrm{eg}: 6 * 6)$ dimension's.

Input image $*$ filter $(3 * 3)=$ result

Filters can be used on an image for distortion, polishing, feature extraction, noise removal and more to help the machine learn a picture's unique characteristics.

result $=\left[\left(n_{i p}+2 p_{p}-c\right) / s\right]+1$

where $\mathrm{n}_{\mathrm{i} / \mathrm{n}}:$ no of input features, $\mathrm{n}_{\mathrm{o} / \mathrm{p}}:$ no of output features, $\mathrm{c}:$ convolution kernel size , p: convolution padding size, s: convolution stride size.

Max - pooling: Input from (2) is taken as z to,

$\mathrm{g}(\mathrm{z})=\max (0, \mathrm{z})$

This obtains an output of size $2 * 2$ matrix.

Then the Flattening layer, where output from (3) is given into Feature vector that can be fed into a classifier or neural network. 
Fully Connected layer: Gives output from the flattering layer into a neural network. The Final output is classified result.

\section{Experimental results}

The HAM10000 image dataset of skin [18] has been used for experimental analysis. The keras model optimizers are selected to be compared, here we have compared between adam, SGD, adadelta and rmsprop optimizers. The Figure 3 and Figure 4 shows the accuracy and loss variation at each epoch, an average of five epochs were compared between each optimizers.

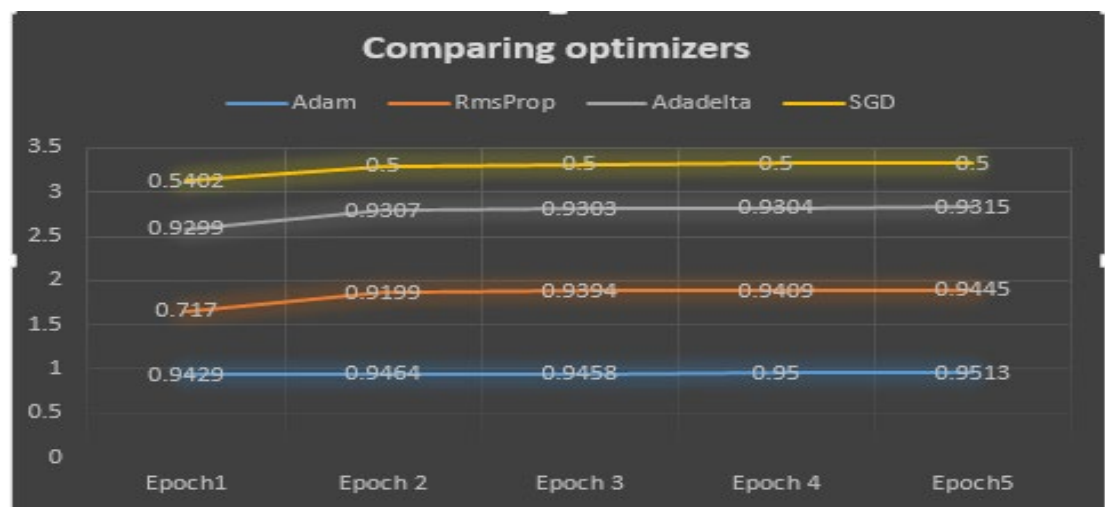

Figure 3: Model optimizer accuracy comparison

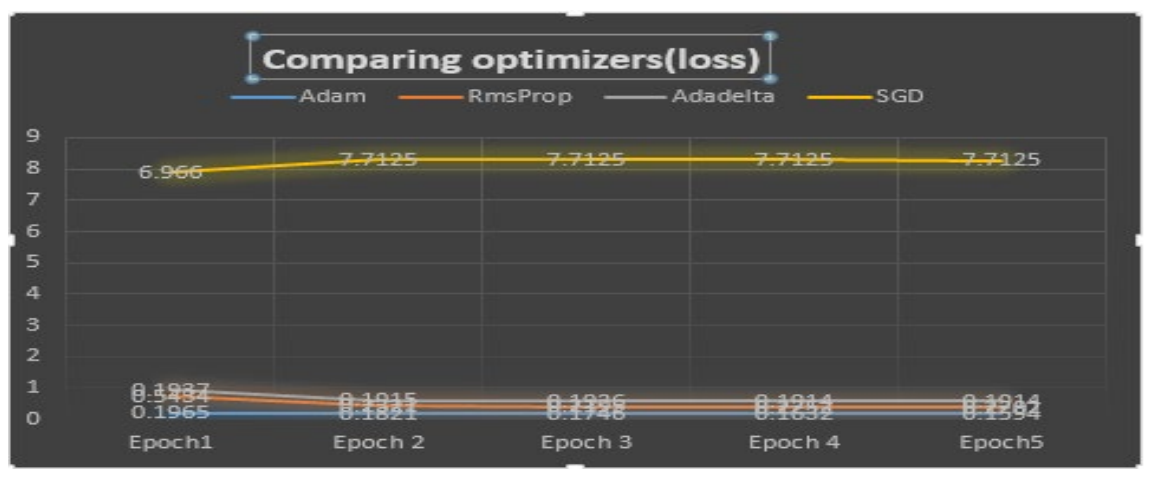

Figure 4: Model optimizer loss comparison

The sample data is divided into set of training data and validation data. Here, the keras and tensorflow framework is being used to build our convolution neural network. Initially for building the neural network we have used the sequential class from keras. After building the $\mathrm{CNN}$, the model is then compiled with adam 
optimizer which seemed to give higher accuracy comparatively and the binary cross entropy as the loss function to calculate the validation and accuracy loss. While training and evaluating the model for accuracy, initially tested with five epochs which gave a accuracy of $95 \%$ and loss of $15 \%$. While tried with higher number of epoch the trained model was able to give almost the same values that were observed for the initial set of epochs. In order to determine the model loss and model accuracy corresponding graphs were plotted. To determine the model accuracy, the graph was plotted with accuracy and validation accuracy as $\mathrm{x}$ and $\mathrm{y}$ axis. Similarly for determining the model loss, the graph was plotted with loss and validation loss as the $\mathrm{x}$ and $\mathrm{y}$ values respectively. While observing the graphical results, its clear that our model is neither over fitting or under fitting. So at any case would be capable to classify if any new image is given to the neural network instead of the image from the dataset. Figure 5 and 6 shows the comparison of accuracy and loss for training and validation set.

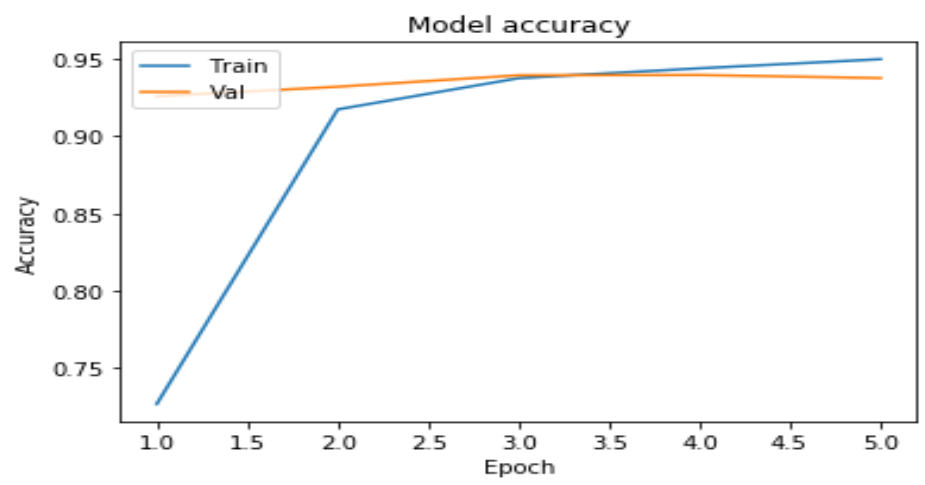

Figure 5: Comparison of Accuracy 


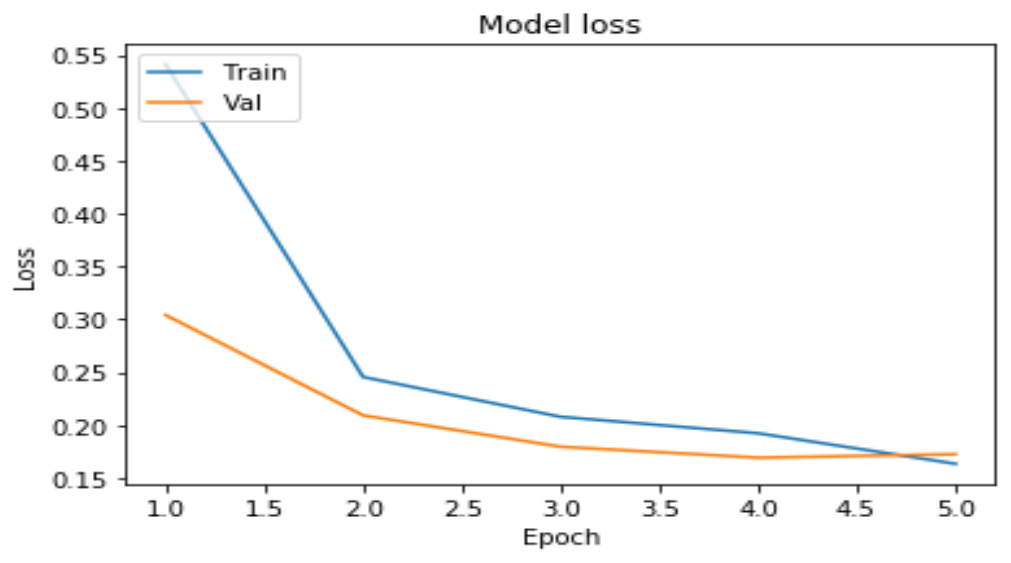

Figure 6: Comparison of loss

\section{Conclusion and future work}

In this work, deep learning was figured out in pervasive health care. The CNN model was assembled and trained to classify imagesof skin. Also in this model various optimizers were compared and the optimizer with high accuracy was employed in the model. The novel aspect involved is the contrast of optimizers and the use of the high frequency optimizer here. This model fits very well with a limited number of images and has been reviewed. How the precision depends on the number of epochs has also been calculated in order to identify potential over suitable issues. It was also decided that 10 epochs were necessary for the model to be successfully educated. The future role will be to attempt and try and adapt this model to realistic tasks on more data sets. More Epochs should also be checked to see if higher performance can be achieved in different problems.

\section{References}

[1] Ms.Poonam T.Handge, Ms.Arti S.Khalkar, MS. Kajal S.Randhe, Ms Pallvi G. Patil, Mr. Deepak Y.Thorat "Skin disease diagnosis system using image processing" 2019

[2] Loddo, Andrea, Cecilia Di Ruberto, and Michel Kocher. \&quot;Recent Advances of Skin Disease Detection Systems Based on Mathematical Morphology.\&quot; Sensors 18, no. 2(2018): 513

[3]Danilo Barros Mendes, Nilton Correia da Silva, "Skin Lesions Classification Using Convolutional Neural Networks in Clinical Images" 2018 
[4] R.s gound,Priyank S.gadre,Jyoti B. Gaikwad,Priyanka K.Wagh "Skin Disease Diagnosis System using Image processing and Data Mining” January2018

[5] Shashi Rekha G, Prof.H. Srinivasa Murthy, Dr.sudarson Jena "Digital DermatologySkin Disease Detection model Using Image Processing” July 2018

[6] Nikita Raut, Aayush Shah, Shail Vira, Harmit sampat "A study on different techniques for skin cancer detection" September 2018

[7] Mrs S.Kalaiarasi,Harsh Kumar,Sourav Patra "Dermatological Disease Detection using Image Processing and Neural Networks" 2018

[8] Qusay Kanaan Kadhim "Classification Of Human Skin Diseases using Data Mining" January 2017

[9] Er.Shrinidhi Gindhi,Ansari Nausheen,Ansari Zoya,Shaikh Ruhin "An Innovative Approach For Skin Disease Detection Using Image Processing and Data Mining" April 2017

[10] Rahat Yasir, Md Ashiqur Rahman, Nova Ahmed "Dermatological disease detection using image processing and artificial neural network" 2014

[11] S.Kolkur, D. Kalbande, P.Shimpi, C.Bapat, J.Jatakia "Human skin detection using RGB $<$ HSV and YCbCr color models" 2017

[12] D.s Zingade,Manali Joshi,Viraj Spare,Rohan Giri "Skin disease detection using artificial neural network" December 2017

[13]Manish Kumar and Rajiv Kumar "An Intelligent System to diagnosis the skin disease" Octobar 2016

[14]Renatageorgia; marthapaes;wouter wan enampt;Marcel brewer; Anna vilanova-"Visual analytics for the exploration of multiparametric cancer imaging" 2015

[15] Dr Rod J Hay, DM, FRCP, Belfast, "Epidemiology and Management of Common Skin Diseases in Children in Developing Countries" 2006

[16] Li-sheng Wei "Skin Disease Recognition Method Based on Image Color and Texture Features" 2005

[17] Berend jan van der Zwaag, Kees Slump and Lambert Spannenburg Analysis of Neural networks for Edge Detection in Proceedings IEEE Benelux Signal Processing Symposium,2002, Swedan

[18] Tschandl P, Rosendahl C, Kittler H. The HAM10000 dataset, a large collection of multi-source dermatoscopic images of common pigmented skin lesions. Scientific data. 2018 Aug 14;5:180161.

[19] Vinod Jagannath Kadam, Shivaji rao Manik rao Jadhav, K.Vijayakumar, "Breast Cancer Diagnosis Using Feature Ensemble Learning Based on Stacked Sparse Auto encoders and Soft max Regression", Image \& Signal Processing, springer, june 2019.

[20] K. Vijayakumar , K. Pradeep Mohan Kumar ,Daniel Jesline, "Implementation of Software Agents and Advanced AoA for Disease Data Analysis", journal of medical systems, Part of Springer Nature 2019. 